\title{
A stable multiply twinned decahedral gold nanoparticle with a
}

\section{barrel-like shape}

Ulises Santiago ${ }^{I}$, J. Jesus Velázquez-Salazar ${ }^{1}$, John Eder Sanchez ${ }^{1}$, Francisco Ruiz-

Zepeda $^{l, \grave{H}}$, José Eduardo Ortega ${ }^{l}$, José Reyes-Gasga ${ }^{2}$, Lourdes Bazán-Díaz ${ }^{1,2}$, Israel

Betancourt $^{1,+}$, Edgar F. Rauch ${ }^{3,4}$, Muriel Veron ${ }^{3}$, Arturo Ponce $^{1}$, and Miguel José-

Yacamán $^{l} *$

${ }^{1}$ Department of Physics and Astronomy, University of Texas at San Antonio. One UTSA

Circle, San Antonio, TX 78249, USA.

${ }^{2}$ Instituto de Física, UNAM. Circuito de la Investigación s/n. Col. Ciudad Universitaria,

Coyoacán, México D. F., 04510. Mexico.

${ }^{3}$ Univ. Grenoble Alpes, SIMAP, F-38000 Grenoble, France

${ }^{4}$ CNRS, SIMAP, F-38000 Grenoble, France

*Corresponding author: Miguel José-Yacamán

Email: miguel.yacaman@utsa.edu

Tel.: +1 210-458-6954

\footnotetext{
${ }^{\dagger}$ Present address: National Institute of Chemistry, Hajdrihova 19, 1000 Ljubljana, Slovenia.

$\$$ On Sabbatical leave from Instituto de Investigaciones en Materiales, Universidad Nacional Autónoma de México, C.P., D.F 04510 México.
} 


\begin{abstract}
In the present work we report a modified configuration of a multiply twinned decahedral barrel-like nanoparticle. The nanoparticle is stabilized by a selective chemical etching, which yielded a truncated shape with multiple high-index planes on the surface. The surface planes and the shape of the nanoparticle have been characterized using electron microscopy techniques, including scanning and transmission electron microscopies and electron diffraction. Coherent electron diffraction revealed streaked Bragg reflections which are in agreement with the high-index facets measured from electron microscope projected images. Crystal orientation mapping assisted by precession electron diffraction was performed under nano-beam conditions giving information about the structure from different orientations. The combination of these techniques allowed us to resolve the 3D structure of the modified nanoparticle and dismiss any possible ambiguities from the interpretation of the projected images in direct space.
\end{abstract}

Keywords: decahedral barrel-like nanoparticle, coherent electron diffraction, crystal orientation maps, precession electron diffraction. 


\section{Introduction}

In the present state of the art on nanotechnology the full control of shape, size and crystal structure of nanoparticles is a fundamental aspect to develop practical applications. In order to achieve the desired structure with also high yield and reproducibility is necessary to fully understand the factors that define the growth of nanoparticles. The growth kinetics results in different shapes, in this way there is an interplay between particle total energy and the kinetics of the growth. Their stability depends mainly upon the size range desired. Different shapes can be produced during growth by balancing the energies binding within the nanoparticle and the ones at the surface planes, where the free energy plays a crucial role in the stabilization of the nanoparticles, and hence giving them their final shape [1-2]. In the smallest size range, nanoparticles and clusters are stabilized by the use of passivating ligands depending on the number of atoms. The diameter of these nanoparticles ranges from few atoms up to few nanometers [3-5]. The next size range includes tens of nanometers in diameter. In this range, plasmonic effects start to appear and the shape can be mostly controlled by kinetics. A prime example being the nanorods, nanowires and hollow particles [6-7]. The next size interval includes larger particles (>100 nm). In this case the shapes are mostly polyhedral nanoparticles based on the Wulff construction formalism [6,8], which has been adapted to multiply twinned particles and oxide nanoparticles [9-10] with recent modifications by Ringe et al [11-12]. In addition, other reports also consider the edge energies obtained by atomistic calculations to determine and predict some shapes as function of the number of atoms distributed along the edges [13-14]. The way particles become stable is by faceting, allowing the formation of structures such as decahedra or icosahedra structures even on this size interval [15-16]. In the present work 
we investigate the shape of a modified truncated decahedron which exhibits a barrel-like shape with multiple facets using single particle diffraction and precession electron diffraction. This serve as a prime example of the very complex structures that can be produced by faceting.

\section{Methods}

The nanoparticles have been prepared by a controlled chemical synthesis following the polyol method. Initially, an ethylene glycol (EG) solution containing polyvinylpyrrolidone (PVP) (0.209 g, Mw = 55000) was heated to boiling, into which an AgNO3 (0.138 mL, 0.6 $\mathrm{mM}$ ) solution in EG was added rapidly under magnetic stirring. After 3 min, aliquots of a HAuCl4 3H20 (0.162 mL, 2.5mM) solution were added each 7 seconds drop by drop. The solution turned to red and the finally changed to reddish brown, indicating precipitation of gold. Then, the mixture (growth solution) was stirred for 10 min more and it was quickly cooled to room temperature. The colloidal solution was washed with ethanol and acetone several times to remove excess of PVP and EG: $1 \mathrm{ml}$ of gold nanoparticles was added to 11 $\mathrm{mL}$ of acetone and the nanoparticles were collected by centrifugation at $6000 \mathrm{rpm}$. Products were dispersed in $3 \mathrm{~mL}$ of ethanol and $8 \mathrm{~mL}$ of acetone and precipitated one more time by centrifugation. This process is repeated several times. The final product of gold nanoparticles was dispersed in ethanol. Our method produces different truncated nanoparticles such as decahedra, cuboctahedra, plates and nano-rods all of them produce facets with low-energy $\{111\}$ surfaces, which are produced by the etching during the chemical synthesis and are in agreement with the surface faceting reported in our previous 
work [14]. The truncated decahedron shape is herein analyzed. An example of the obtained nanoparticles with different shapes is shown in Figure S1 of supplemental material.

A drop of the colloidal solution was deposited onto copper holey carbon grids for characterization. The analysis of the shape and projected planes of the nanoparticles was performed by scanning electron microscopy (SEM) and transmission electron microscopy (TEM). The SEM images were obtained using a field emission gun scanning transmission electron microscope Hitachi S5500 operated at $30 \mathrm{kV}$. The TEM imaging information was obtained using field emission gun JEOL microscopes, 2010F and 200F ARM both of them operated at $200 \mathrm{kV}$. In the present work we determine the geometry of modified gold decahedra nanoparticles by using selected area electron diffraction (SAD), precession electron diffraction-assisted automated crystal orientation mapping (PED ACOM-TEM) [17] and coherent electron diffraction (CED) $[18,19]$. These electron diffraction techniques provided useful information about the crystalline structure and surface plane facets of the barrel-like nanoparticle. To perform PED and CED, a small area is irradiated under nanobeam electron diffraction conditions, using the smallest condenser aperture and reducing the diameter of the electron irradiation to a few nanometers. In PED we used a probe size of around $1.5 \mathrm{~nm}$ in diameter, while in CED, in order to illuminate the whole particle, the probe size was slightly larger than the nanoparticle size (more than $100 \mathrm{~nm}$ ). A CED pattern shows streaks lines in the Bragg reflections; these streak lines are measured with respect to the Bragg reflection and are related to high index crystallographic planes showing directions normal to the facets of the nanoparticle. These streaks come from the intersection of the Ewald sphere with reciprocal lattice rods of the nanoparticle facets. The intersection is almost tangentially when the facet is normal to the incident electron beam. The sharper the facet the longer the length of the streaks [19]. On the other hand, PED 
ACOM-TEM gives the full crystallographic information of the object at a specific area of the region of interest (ROI), by providing a crystallographic map at the nanometer scale and making easier the identification of crystalline phases in semiconductor materials, metallurgy and nanostructured materials [20-22]. In the ACOM-TEM technique the electron beam is scanned across the sample and collects the electron diffraction patterns using an ultrafast charge-coupled device (CCD) camera attached to the viewing screen of the microscope. All of the electron diffraction patterns collected are then indexed using an automatic matching procedure, identified by a cross-correlation with preselected theoretical templates from a database. The ACOM-TEM technique is assisted by PED to enhance the quality of the diffraction pattern matching, since PED reduces the dynamic effects arising in electron diffraction [23]. PED experiments were conducted using Nanomegas hardware and software package [24]; further details of the experimental set up and from the analysis process are available in the supplementary material.

\section{Results and Discussion}

The truncated decahedral Au nanoparticles exhibit a barrel-like form where the base is pentagonal in shape (similar to a truncated decahedral particle) and the lateral view is formed by 50 planes faceted one to each other 5(sides)x2(bipyramid $)(2 x\{320\}+2 x\{221\}+1 x\{111\})$. Figure 1 shows the scanning electron microscopy images of the nanoparticle. These facets stand the body at an angle of 143 degrees to the base; the identified planes are labeled in Figures 1a and 1b. Table 1 presents the theoretical $\{h k l\}$ planes and the measured angles from the SEM images of Figure 1. It is worth noting that in the drawing of Table 1 the particle is seen along the direction [110], and that, e.g. the 
$\{320\}$ and $\{221\}$ facets are not parallel to [110]; in fact $\{221\}$ facet is $13^{\circ}$ of inclination with respect to [110], about the same goes with $\{320\}$. However, despite this inclination, the angle between the facets are practically the same. That is, the angles in table 1 could varied 1 or $2^{\circ}$ from the observed value, but they will be very close to the calculated value.
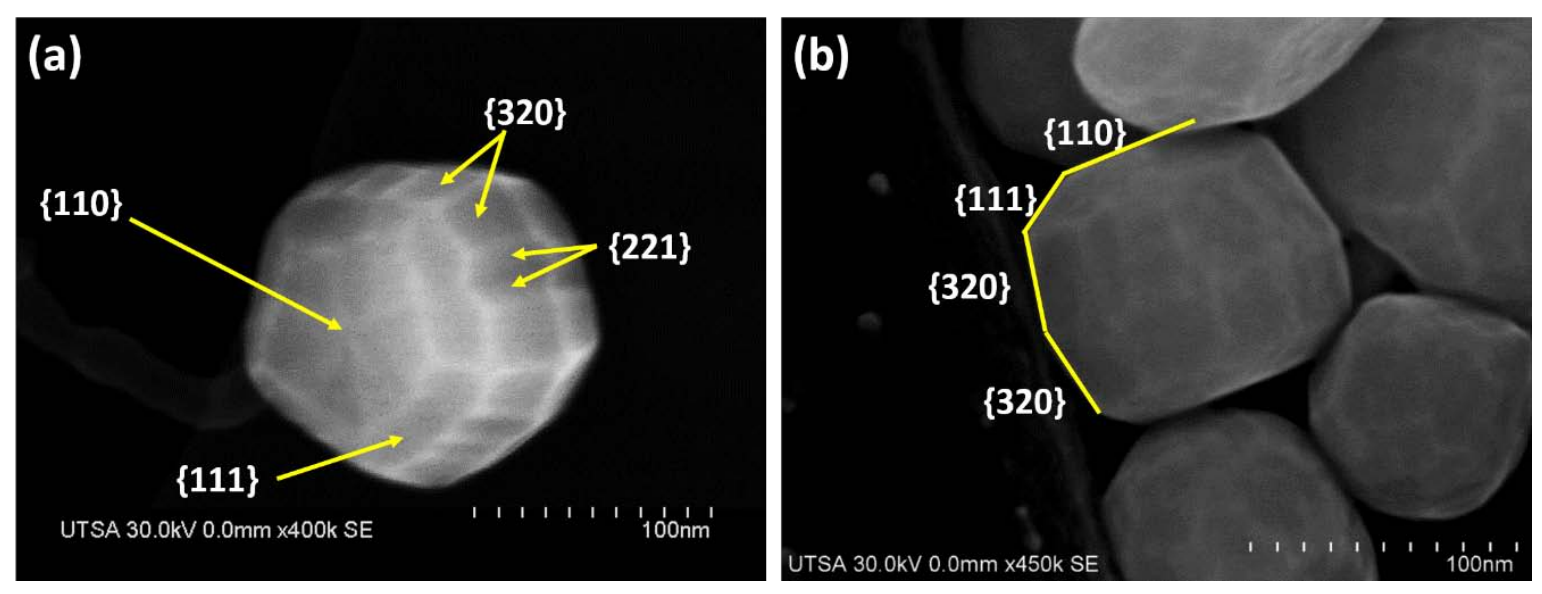

Figure 1. SEM images of the nanoparticle. a) Front view; b) side view. The arrows in (a) indicate the surface planes of the surface nanoparticle. The yellow lines in (b) indicate the edge surface.

TEM imaging has been used to confirm the angles measured in SEM images. Within the nanoparticle the atomic distribution correspond to a regular multiple twin decahedral structure. In Figure 2, TEM images are shown in two different orientations: along the $<110>$ direction and along perpendicular to the $<110>$ (the side view direction). The fcc unit cell of $\mathrm{Au}$ has been taken as the reference system. The side view in Figure 2a shows the projected planes $\{320\},\{111\}$ as well as the truncated plane of the decahedron $\{110\}$. Angles are consistent with those measured in the Figure 1; in Figures $2 \mathrm{~d}$ and $2 \mathrm{e}$ we have measured the angles on the particle oriented close to the $<110>$ zone axis. Especially, 
Figure 2e shows the contours of the nanoparticle pictured in Figure $2 \mathrm{~d}$ in order to measure more precisely the angles of the facets in the nanoparticle. In Figure $2 b$, the corresponding SAD pattern from the side view of the particle (Figure 2a) is shown.

Table 1. Interplanar angles measured for the barrel-like nanoparticle and compared with their theoretical values.

\begin{tabular}{|c|c|c|c|c|}
\hline \multirow{2}{*}{ Projection } & \multirow{2}{*}{$\begin{array}{c}\text { Interplanar } \\
\text { angles }\end{array}$} & \multirow{2}{*}{$\begin{array}{c}\text { Planes } \\
\left\{\mathbf{h}_{1} \mathbf{k}_{\mathbf{1}} \mathbf{l}_{1}\right\} / /\left\{\mathbf{h}_{2} \mathbf{k}_{2} \mathbf{l}_{2}\right\}\end{array}$} & \multicolumn{2}{|c|}{ Angles (in degrees) } \\
\hline & & & Theoretical & Experimental \\
\hline & & $\left\{\begin{array}{lll}3 & 2 & 0\end{array}\right\} / /\left\{\begin{array}{lll}3 & 2 & 0\end{array}\right\}$ & 133.81 & $135 \pm 3$ \\
\hline (1110) & & $\left\{\begin{array}{lll}2 & 2 & 1\end{array}\right\} / /\left\{\begin{array}{lll}2 & 2 & 1\end{array}\right\}$ & 152.73 & $155 \pm 3$ \\
\hline & & $\left\{\begin{array}{lll}3 & 2 & 0\end{array}\right\} / /\left\{\begin{array}{lll}2 & 2 & 1\end{array}\right\}$ & 157.59 & $156 \pm 2$ \\
\hline & & $\left\{\begin{array}{lll}3 & 2 & 0\end{array}\right\} / /\left\{\begin{array}{lll}1 & 1 & 1\end{array}\right\}$ & 143.19 & $140 \pm 3$ \\
\hline 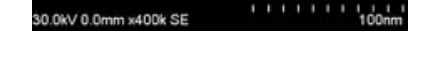 & & $\left\{\begin{array}{lll}1 & 1 & 0\end{array}\right\} / /\left\{\begin{array}{lll}1 & 1 & 1\end{array}\right\}$ & 144.74 & $144 \pm 2$ \\
\hline SEM Micrograph from Figure 1a & & & & \\
\hline
\end{tabular}

The spots in the SAD pattern have been identified as a combination of two overlapped zone axes $[001]+[112]$. The overlapping of these two zone axes is simulated in Figure 2c and correlates well with the reflections observed in the experimental SAD pattern of the Figure $2 \mathrm{~b}$. The extra spots that appear in Figure $2 \mathrm{~b}$ arise from the combination of double diffraction due to the multiple twinned structure and the nano size of the particle $[7,25]$. It is well known that the pattern in Figure $2 \mathrm{f}$ is the result of the convolution of five [110] 
patterns each one rotated by $72^{\circ}$. The $\{221\}$ planes projected from the side view are not totally symmetric in the five sides of the decahedron due to a slightly tilting of the particle out of the $<110>$ twinning axis.
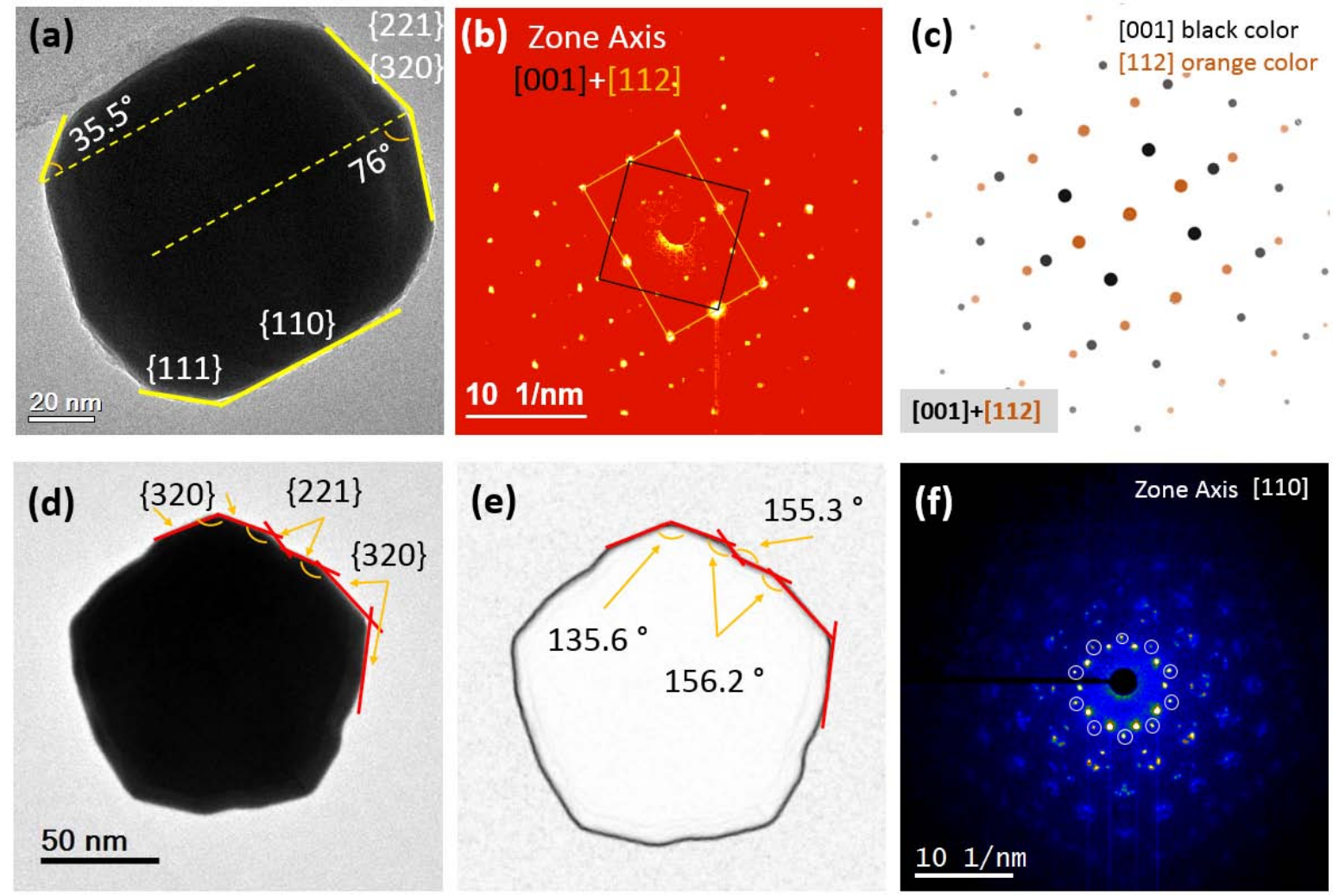

Figure 2. TEM images from (a) side view, (d) close to the five-fold (along $<110>$ direction) and (e) contours filtered from (d). Projected planes and angles are measured from both orientations. SAD patterns in (b) and (f) are recorded along the side view and close to the five-fold, respectively. The circled diffraction spots in (f) correspond to the $\{002\}$ planes. (c) Simulation of two overlapped electron diffraction patterns [001] + [112].

Once the relationship between the axis [110] and the angle is known in the truncated decahedral nanoparticle, it is possible to know which planes are observed when the particle is seen side view. In the cubic system, the direction $[h k l]$ is perpendicular to the plane 
$(h k l)$ and given that the observation axis is along one of the $\{110\}$ axes family, the diffraction pattern along the [110] zone axis is used to calculate these planes. Using solely SAD, the high index faceting cannot be identified with ease; in such cases, CED has demonstrated to be a powerful tool for the $3 \mathrm{D}$ interpretation of high index facets in polyhedral nanoparticles and other nanostructures with atomic steps on their surfaces [18, 19]. Following this line of thought we have performed CED in two directions as shown in Figure 3. The CED pattern in figure 3a shows an inset of the illumination conditions for the CED method along the five-fold direction. A similar image is shown in Figure $3 b$ for the nanoparticle oriented slightly out of the five-fold direction. In both cases we have obtained streak lines which measured angles revealed facets also identified in images from Figures 1 and 2. For instance, the CED pattern in Figure 3a shows streak lines oriented at $54^{\circ}$ with respect to the $<111>$ Bragg reflection. If we consider the particle oriented along $<110>$, and taking the cubic system as of reference, the planes $\{111\}$ are inclined more than the planes $\{320\}$ and $\{221\}$ from the side part of the nanoparticle. That is, directions [221] and [320], which are normal to the planes $\{221\}$ and $\{320\}$ respectively, made an angle of about $13^{\circ}$ with the $[110]$ axis, approximately, while the angle between planes $\{111\}$ and $\{110\}$, or directions [111] and [110], it is of $32^{\circ}$, approximately. This is what is observed in the produced the streak lines. These $54^{\circ}$ multiplied 10 times gives the sum of the inner angles in a pentagon. The pattern in Figure $3 \mathrm{~b}$ corresponds mainly to the [111] zone axis with some contributions of the [112] zone axis which produces the streak lines for the faces $\{320\}$ and $\{221\}$. This was also confirmed by electron tomography from which we performed $3 \mathrm{D}$ reconstruction and visualization using the algebraic reconstruction technique (ART) [26]. The details about the electron tomography and 3D reconstruction are available in the supplementary material as well as the video of the $3 \mathrm{D}$ reconstruction. 

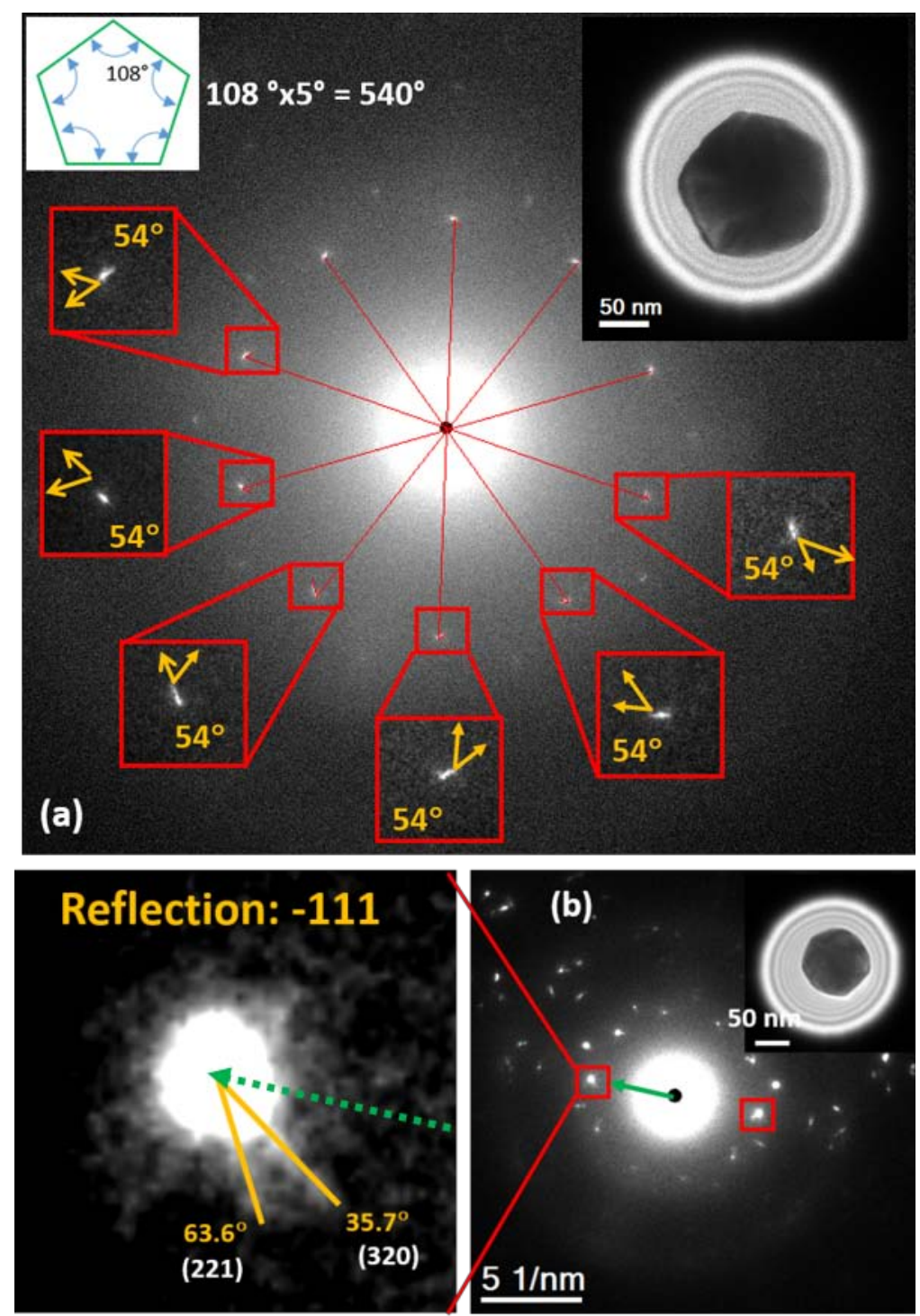

Figure 3. Coherent electron diffraction patterns from the truncated decahedral particle. (a) Along plan-view; (b) along side-view. The pattern in (a) is the result of the convolution of five [110] patterns rotated by $72^{\circ}$ each other. The yellow arrows correspond to the direction of reference, taken from the transmitted beam, and to the direction of the streak, yielding $54^{\circ}$ between them in all cases. The pattern in (b) corresponds to the [111] zone axis together with some spots of the [112] zone axis electron diffraction pattern.

The analysis of the geometry of these nanoparticles has been performed using the PED ACOM-TEM technique. A full set of diffraction patterns was collected while the 
precessing electron beam was scanning the ROI. The step size used in the scanning was 1.5 $\mathrm{nm}$, about the same as the probe size. The results of the indexation are later displayed as a crystal orientation map of the ROI. In Figure 4, the barrel-like nanoparticle has been oriented along the five-fold symmetry. Each orientation (colored) map, refers to a particular viewing direction, i.e. the color is assigned based on the crystal orientation along this particular direction. Experimental details about the PED ACOM-TEM measurements are available in the supplementary material. Figure 4 shows also the color code map for the crystal directions (Figure 4b) and a model of the five-fold nanoparticle orientation, the axes and e-beam directions (Figure 4a). In Figure S3 of the supplementary material an example of the matching between a particular pattern and the relevant template is shown. Figures 4c, $4 \mathrm{~d}$ and $4 \mathrm{e}$ show the orientation of each part of the decahedron observed from the three main reference directions $\mathrm{x}, \mathrm{y}$, and $\mathrm{z}$, respectively. Figure 4c shows the planes $\{111\}$ (blue color) and the $\{110\}$ (green color) which are consistent with the view using as a reference the " $\mathrm{x}$ " axis. Note that in Figure $4 \mathrm{~d}$ all crystals show the same color, this is clearly expected because the orientation of the nanoparticle is such that the viewing direction (e-beam direction) is parallel to the common [110] direction. The colors displayed in the orientation maps of Figure 4, correspond to the [111], [001] and [101] reference directions and to the [112], [113] and [212] directions, having all crystals an orientation of $72^{\circ}$ between them. 


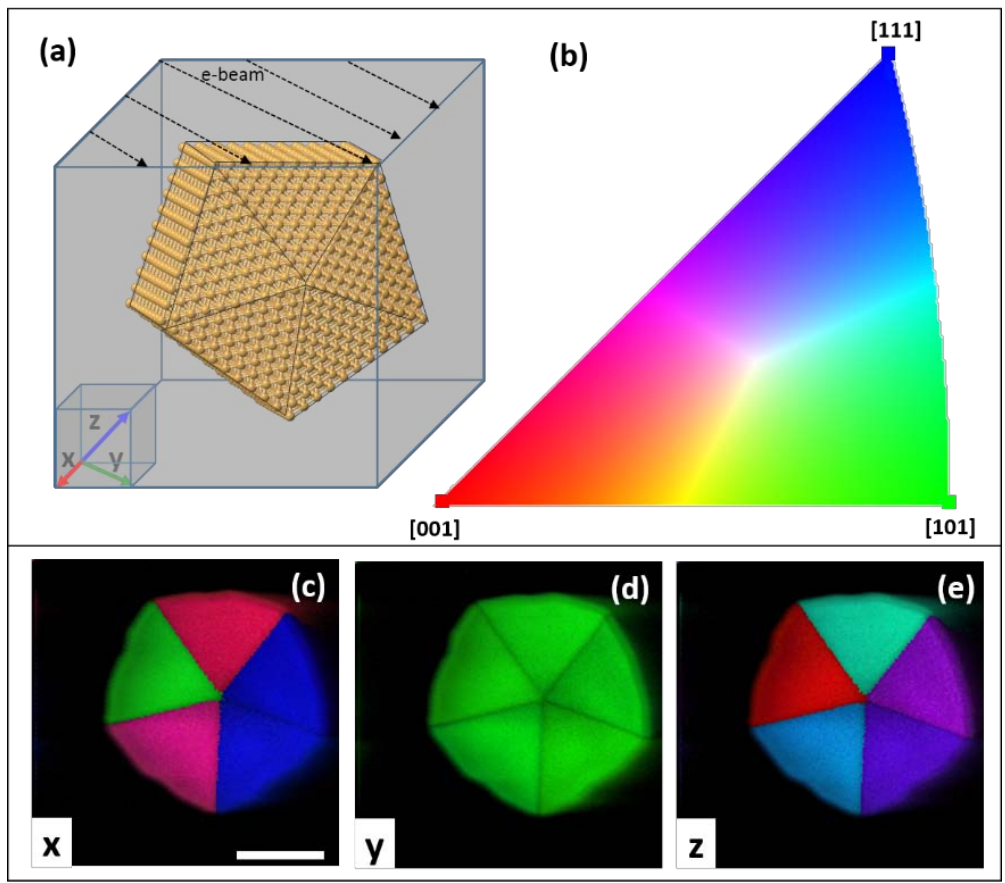

Figure 4. (a) Decahedron model and the frame of reference for the crystal orientation maps.

(b) Color code inverse pole figure taken from the inverse pole diagram obtained along the [001] direction (included in Figure S3). Crystal orientation maps corresponding to $\mathrm{x}, \mathrm{y}$ and $\mathrm{z}$ directions, (c), (d) and (e) respectively (scale bar is $50 \mathrm{~nm}$ ).

In addition, we have recorded crystal orientation maps on two different tilt directions, 50 degrees away from each other. First, the particle analyzed was tilted close to a side view at +25 degrees from a starting point. In this orientation (Figure 5-top set) the five crystals are identified and indicated by different colors in at least two of the orientation maps, but the interfaces are very poorly defined. This is because the common [110] axis is inclined $60^{\circ}$ with respect to the beam direction (z-direction). Consequently, crystals are massively overlapping in the thickest part of the nanoparticle and the selection of the predominant diffraction pattern is confused by the strong signal related to the additional patterns. However, the twin relationships have been confirmed in all cases by computing the 
disorientation between two adjacent crystals $\left(60^{\circ}\right)$ and the associated rotation axis $([111])$. A second scan was performed after tilting the sample $50^{\circ}$ around the sample holder axis (Figure 5 - bottom set). In this new orientation, the common [110] axis is far closer from the incident beam direction $\left(20^{\circ}\right)$ and overlapping artifacts are reduced. In particular, there is a substantial improvement in crystal interfaces localization. Again, the five crystals are recognized and the twin characters confirmed. Note that for figure 5 the reference axes have been tilted $44^{\circ}$ clockwise. This is in order to represent the orientation in the physical reference frame related to the sample holder. Obviously, the crystallographic orientations along $\mathrm{x}$, that is, along the sample holder main axis, should not be altered with the tilt angle. Indeed, the colors in the orientation map labeled ' $\mathrm{x}$ ' remain unchanged for every crystal before and after tilting (compare Fig. 5c and c') leading to an easier interpretation of the results.

To gain a better view of the $3 \mathrm{D}$ configuration of the nanocrystal, correlation contours are added in figures 5.f and 5.f'. These contours corresponds to the average value of the crosscorrelation indexes between a given diffraction pattern and the surroundings ones. A large value (white) is obtained when the diffraction patterns are similar, i.e. within a crystal. The value decreases if a change in the diffracting signal is noticed. Dark lines are expected to highlight grain or phase boundaries. The contrast is enhanced when the boundaries are aligned with the beam direction (abrupt change of diffraction patterns). In the present case, the inner twin boundaries are detected, although not perfectly but sufficiently to guess the orientations of the crystals before and after the tilt. From this views there is clearly no twin cutting the nanoparticle in cross section along the direction [110]. Additional particles with and without multiple twins were tested with the same method and for the case of twinned 
particles the contours are clearly visible. These images are available in the supplementary information Figure S5.

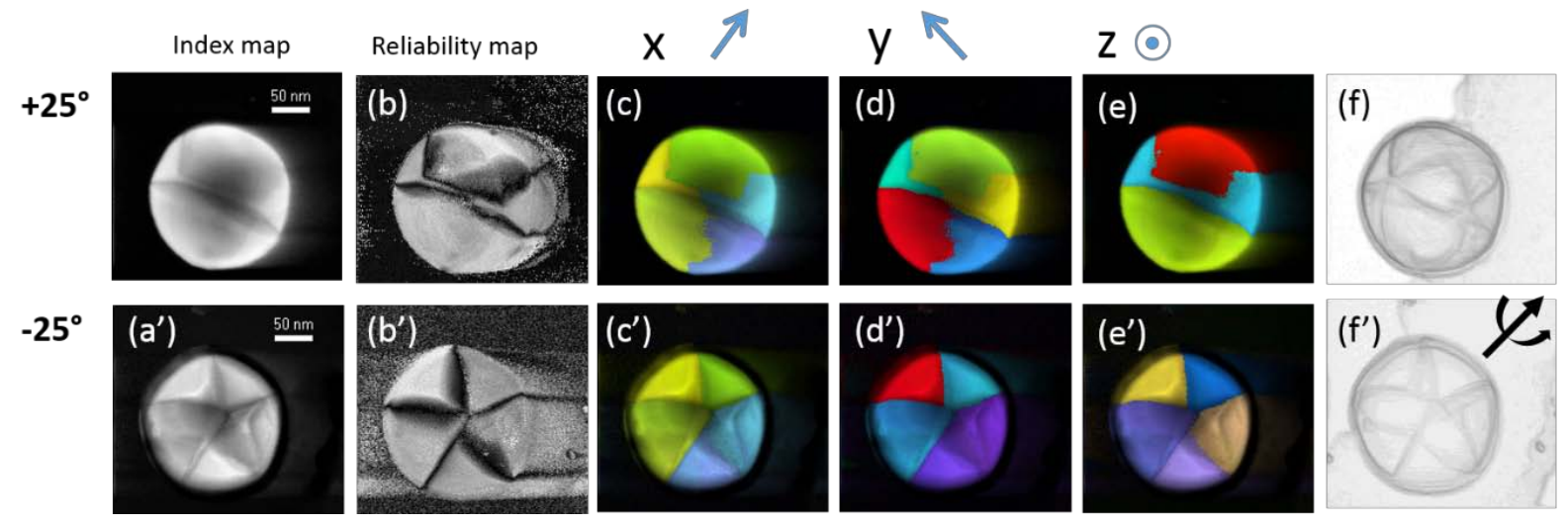

Figure 5. Visualization maps for index, reliability, crystal orientation with reference directions $\mathrm{x}, \mathrm{y}$ and $\mathrm{z}$, and contour representation, obtained for a decahedral barrel-like nanoparticle at two different tilts $+25^{\circ}$ and $-25^{\circ}$ with respect to a starting point.

\section{Conclusions}

We have here reported a modified stable decahedral truncated nanoparticle with a "barrellike" shape. Electron diffraction patterns revealed the facets on its surface and orientation phase maps were used to distinguish individual units within the nanoparticle. The particle has an equivalent pentagonal symmetry with respect to known nanoparticles. The planes of the nanoparticle have been identified by crystal orientation maps using ultra-fast scanning electron diffraction under precession mode and coherent electron diffraction, both diffraction modes not only complement one to each other, but validates and gives consistency to the observed geometry. 


\section{Acknowledgements}

The authors would like to acknowledge the NSF PREM DMR \#0934218, the Department of Defense \#64756-RT-REP and CONACYT \# 250836. I. Betancourt acknowledges financial support from PASPA-DGAPA, UNAM and CONACYT, Mexico, during his sabbatical leave. E.F. Rauch acknowledges to Ákos K. Kiss from University of Pannonia Hungary for suggesting the correlation contour principle prior to the developments shown in the paper. The microscopy work was supported by the National Institute on Minority Health and Health Disparities (NIMHD) in the program Research Centers in Minority Institutions Program (RCMI) Nanotechnology and Human Health Core (G12MD007591).

\section{References}

[1] A.S. Barnard, Clarifying stability, probability and population in nanoparticle ensembles, Nanoscale 6 (2014) 9983-9990.

[2] F. Baletto and R. Ferrando, Structural properties of nanoclusters: Energetic, thermodynamic, and kinetic effects, Rev. Mod. Phys. 77 (2005) 371-423.

[3] M. José Yacamán, J.A. Ascencio, H.B. Liu, J. Gardea-Torresdey, Structure shape and stability of nanometric sized particles, J. Vac. Sci. Technol. B 19 (2001) 1091-1103.

[4] A. Dass, Faradaurate nanomolecules: a superstable Plasmonic 76.3 kDa Cluster, J. Am. Chem. Soc. 133 (2011) 19259-19261.

[5] D. Bahena, N. U. Santiago, A. Tlahuice, A. Ponce, SBH Bach, B. Yoon, RL Whetten, U. Landman, M. Jose-Yacaman, STEM Electron Diffraction and High-Resolution Images Used in the Determination of the Crystal Structure of the Au-144(SR)(60) Cluster, J. Phys. Chem. Lett. 4 (2013) 975-981. 
[6] S. Ino, Stability of multiply-twinned particles, J. Phys. Soc. Jpn. 27 (1969) 941-953.

[7] J. Reyes-Gasga, J. L. Elichiguerra, C. Liu, A, Camacho-Bragado, J. M. MontejanoCarrizales, M. Jose-Yacaman, On the structure of nanorods and nanowires with pentagonal cross-sections, J. Crystal Growth 286 (2006) 162-172.

[8] G. Wulff, Zur frage der Geschwindigkeit des Wachsthum und der Auflosung der Krystallflachen, Kristallogr. Mineral 34 (1901) 449-530.

[9] L.D. Marks, Surface structure and energetics of multiply twinned particles, Philos. Mag. A 49 (1984) 81-93.

[10] J. A. Enterkin, K. R. Poeppelmeier, L. D. Marks, Oriented catalytic platinum nanoparticles on high surface area strontium titanate nanocuboids, Nano Lett. 11 (2011) 993-997.

[11] E. Ringe, R.P. Van Duyne and L.D. Marks, Kinetic and thermodynamic modified Wulff constructions for twinned nanoparticles, J. Phys. Chem. C 117 (2013) 15859-15870.

[12] E. Ringe, R.P. Van Duyne and L.D. Marks, Wulff construction for alloy nanoparticles, Nano Lett. 11 (2011) 3399-3403.

[13] J.C. Hamilton, Edge energies: atomistic calculations of a continuum quantity, Phys. Rev. B 73 (2006) 125447.

[14] G. Casillas, J.J. Velazquez-Salazar and M. Jose-Yacaman, A New Mechanism of Stabilization of Large Decahedral Nanoparticles, J Phys. Chem. C 116 (2012) 8844-8848.

[15] E. Carbo-Argibay, B. Rodriguez-Gonzalez, I. Pastoriza-Santos, J. Perez-Juste and L.M. Liz-Marzan, Growth of pentatwinned gold nanorods into truncated decahedra, Nanoscale 2 (2010) 2377-2383. 
[16] Z. Zhou, N. Tian, Z. Huang, D. Chen and S. Sun, Nanoparticle catalysts with high energy surfaces and enhanced activity synthesized by electrochemical method, Faraday Discuss. 140 (2008) 81-92.

[17] E. F. Rauch, M. Véron, Automated crystal orientation and phase mapping in TEM, Mater. Charact. 98 (2014) 1-9.

[18] J.M. Zuo, M. Gao, J. Tao, B. Li, R. Twesten, I. Petrov, Coherent Nano-area electron diffraction, Microsc. Res. Tech. 64 (2004) 347-355.

[19] A.B. Shah, S.T. Sivapalan, B.M. DeVetter, T.K. Yang, J. Wen, R. Bhargava, C.J. Murphy and J.M. Zuo, High-Index Facets in Gold Nanocrystals Elucidated by Coherent Electron Diffraction, Nano Lett. 13 (2013) 1840-1846.

[20] I. Ghamarian, Y. Liu, P. Samimi, P.C. Collins, Development and application of a novel precession electron diffraction technique to quantify and map deformation structures in highly deformed materials - as applied to ultrafine-grained titanium, Acta Mater. 79 (2014) 203-215.

[21] A. Avilov, K. Kuligin, S. Nicolopoulos, M. Nickolskiy, K. Boulahya, J. Portillo, G. Lepeshov, B. Sobolev, J. P. Collette, N. Martin, A. C. Robins, and P. Fischione, Precession technique and electron diffractometry as new tools for crystal structure analysis and chemical bonding determination, Ultramicroscopy 107 (2007) 431-444.

[22] F. Ruiz-Zepeda, Y.L. Casallas-Moreno, J. Cantu-Valle, D. Alducin, U. Santiago, M. Jose-Yacaman, M. Lopez-Lopez and A. Ponce, Precession electron diffraction-assisted crystal phase mapping of metastable c-GaN films grown on (001) GaAs, Microsc. Res. Tech. 77 (2014) 980-985. 
[23] E. F. Rauch, M. Véron, J. Portillo, D. Bultreys, Y. Maniette, S. Nicolopoulos, Automatic crystal orientation and phase mapping in TEM by precession diffraction, Microsc. Anal. 22 (2009) S5-S8.

[24] Nanomegas Company Home Page. http://www.nanomegas.com/ (accessed: 7, 2015).

[25] J. L. Elechiguerra, J. Reyes-Gasga and M. Jose Yacaman, The role of twinning in shape evolution of anisotropic noble metal nanostructures, J. Mater. Chem., 16 (2006) 3906-3919. [26] C. Messaoudi, T. Boudier, C.O. Sanchez-Sorzano and S. Marco, TomoJ: tomography software for three-dimensional reconstruction in transmission electron microscopy, BMC Bioinformatics 8 (2007) 288. 


\section{Graphical abstract}

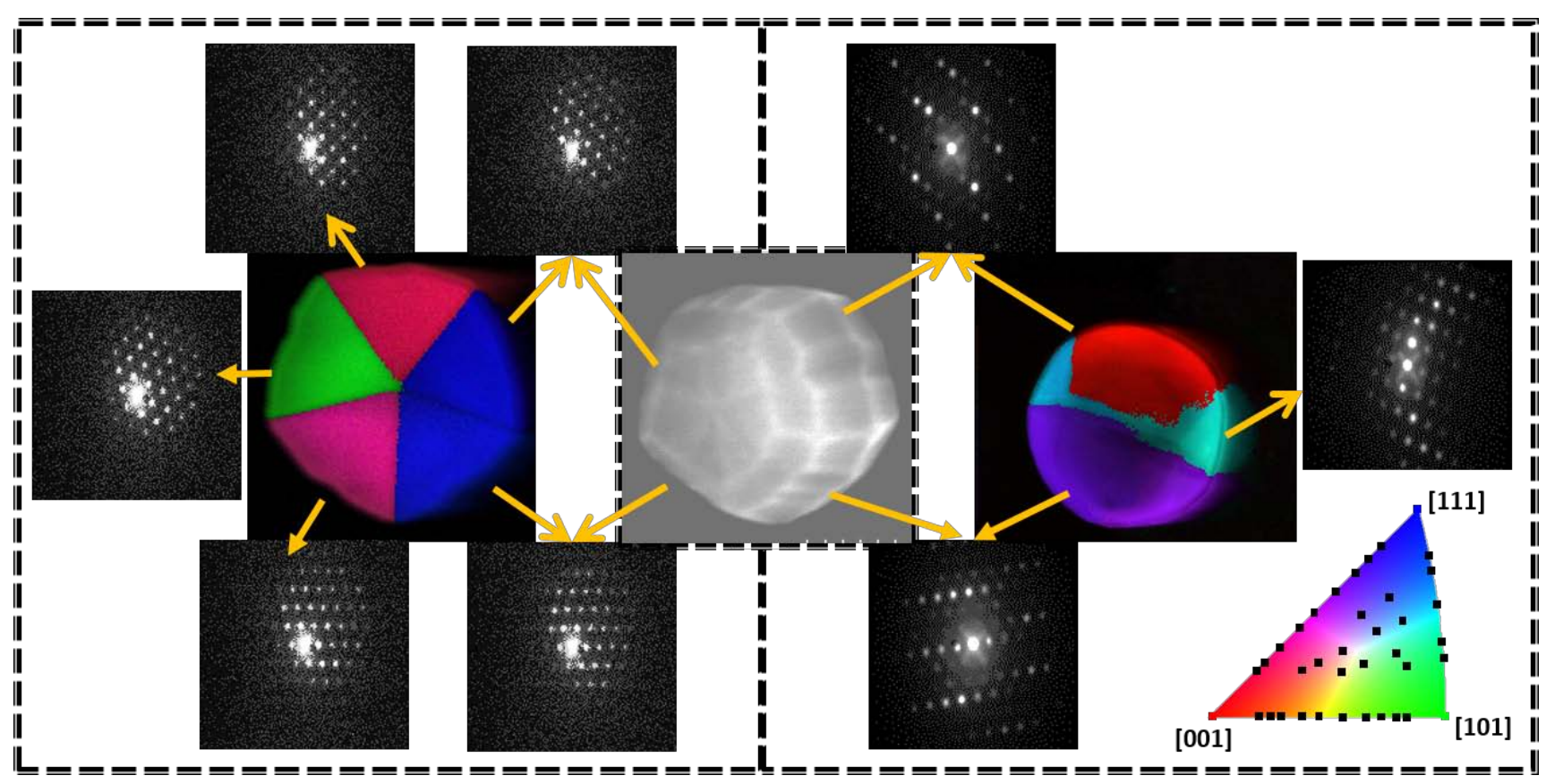

Electron diffraction patterns and crystalline orientation maps of the new multiply twinned decahedral gold nanoparticle with a barrel-like shape. 